\title{
Preliminary Analysis of the Calibration Procedures for a Geodesic Antenna Array (GEODA)
}

Miguel A. Salas Natera, Ramón Martínez, Leandro de Haro Ariet

Radiation Group. Dpto. De Señales, Sistemas y Radiocomunicaciones. Universidad Politécnica de Madrid. ETSI de Telecomunicación.

\{msalasn, ramon\}@gr.ssr.upm.es

\section{Introduction}

Nowadays, earth stations for downloading data from LEO (Low Earth Orbit) satellites make use of large reflector antennas. These antennas pose a number of impairments regarding their mechanical complexity, lower flexibility, network efficiency and higher cost [1]. Furthermore, reflector antennas can track only one satellite at a time, so that the efficiency of the earth segment is reduced [2]. In order to improve the performance of traditional earth stations, the feasibility of other antenna technologies must be evaluated and one alternative are the conformal adaptive antennas based on multiple planar arrays as geodesic antenna array (GEODA) [3]. These antennas have several advantages over large dishes [4] and the efficiency to track several satellites simultaneously is a function of the number of antenna elements and the correct calibration of the antenna arrays. These antennas use adaptive beamforming algorithms with spatial reference to track the satellites, and the compute of a close approach of the spatial reference as well the direction of arrival depends on the calibration algorithm performance. In this paper an analysis of the calibration procedure for smart antennas to be implemented in the GEODA for satellites communications is presented.

\section{Situation for GEODA Smart Antenna Calibration}

The aim of this work is to tackle the implementation of the calibration procedures that must be applied to a GEODA antenna. Since the position of a satellite is known, the most suitable beamforming algorithms are those based on spatial references. Because of that and what was explained before, the precise calibration of the array is an important stage of the GEODA implementation.

\begin{tabular}{|c|c|c|c|}
\hline Simulations & \multicolumn{3}{|c|}{$\begin{array}{l}\text { Parameters (Uniform and Non-uniform array): } \\
\text { - Sensor state (on-off) } \\
\text { - Sensor Magnitude error } \\
\text { - Sensor Phase error }\end{array}$} \\
\hline $\begin{array}{l}\text { Off-line } \\
\text { Calibration }\end{array}$ & \multicolumn{3}{|c|}{$\begin{array}{l}\text { Compensation of several errors: } \\
\text { - Sensor position error } \\
\text { - Sensor Gain hardware error } \\
\text { - Sensor Phase hardware error }\end{array}$} \\
\hline $\begin{array}{c}\text { On-line } \\
\text { Calibration }\end{array}$ & $\begin{array}{l}\text { News Algorithms inputs: } \\
\text { - Entropy measurement } \\
\text { - Digital Signal level } \\
\text { - Sensors control signals }\end{array}$ & $\begin{array}{l}\text { - Sensor temperature } \\
\text { - Digital signal phase }\end{array}$ & $\begin{array}{l}\text { Others algorithms: } \\
\text { - Temporal reference } \\
\text { - Spatial reference } \\
\text { - Look-up table approach }\end{array}$ \\
\hline
\end{tabular}

Figure 1. Flowchart of the calibration procedure

The steps for the development of the calibration algorithm are presented in Figure 1. The process is divided in three sequential stages: first, the simulations of the off-line and on- 
line calibration algorithms must be carried out; second, the implementation of the off-line calibrations based on extensive anechoic chamber measurements is performed; and finally, the on-line calibration is implemented and tested with field trials.

The calibration methods which have been analyzed to carry out the off-line calibration can be separated in two groups of algorithms . The first set of techniques [5][6] are based on the injection of an equal phase signal to every channel of the antenna array in order to process the received signal and obtain the compensation matrix. These techniques address the network mismatch issues. The second [7][8] family of techniques use a signal from known direction transmitted on-site to the antenna array, and then analyze the output of the antenna array in order to obtain the direction of arrival and compute the compensation matrix. These techniques deal with the uncertain sensor locations, the mutual coupling effects, the differences in the gain and phase response of the sensor, and the cabling phase and gain errors.

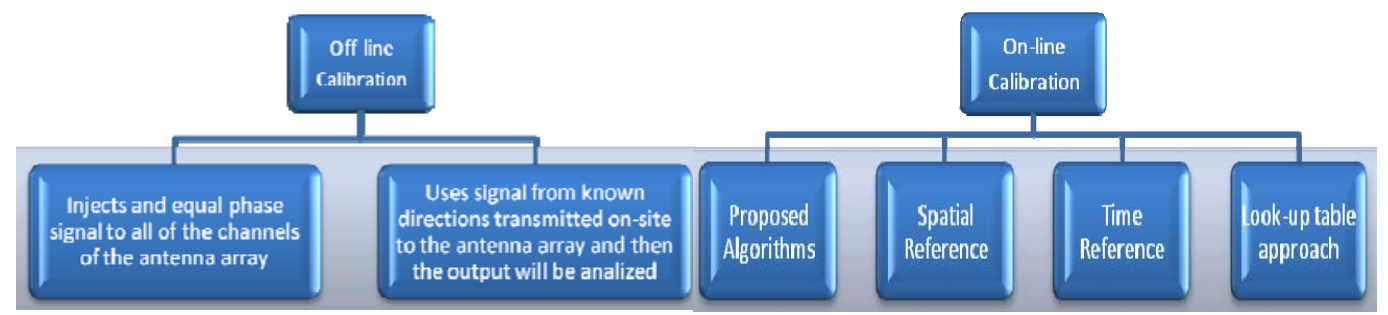

Figure 2. Off-line (left) and On-line (right) calibration methods to be evaluated

In this paper, we propose a new method for the on-line Calibration Smart Antenna System to be implemented in the GEODA antenna for satellite communications and tracking. The aim of the proposed algorithm is the self-calibration of the antenna array continuously, so that this algorithm has to be transparent to the signal processing.

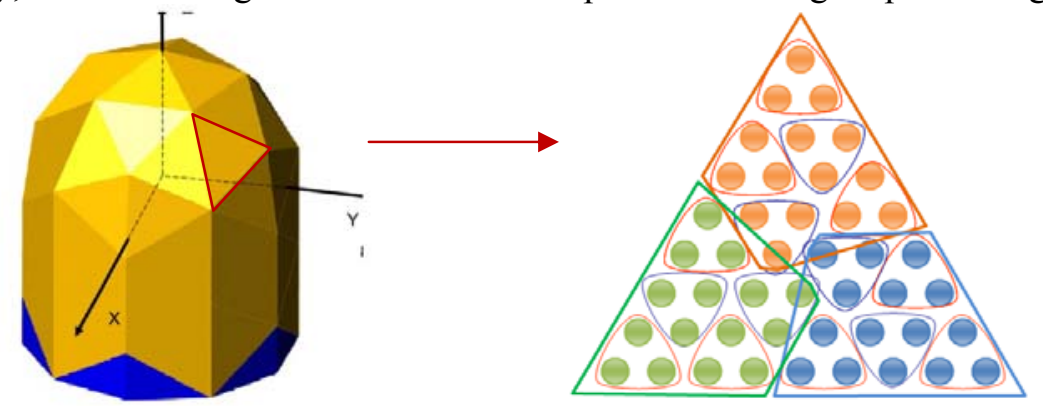

Figure 3. GEODA antenna (left) and Array with 3x15 elements (right)

\section{Simulations}

Simulation result was obtained for array panel of the GEODA showed in Figure 3. In order to quantify the effects of the above mentioned errors on the beamforming process, some simulation results will be presented for two particular scenarios. The Figure 4 shows the effect of having uncompensated sensor locations error and the result is compared in terms of the electric far field pattern of the antenna array. Due to these errors side lobes appear and these add noise and interference to the received signal degrading the reception in a particular direction of the target. The SNR is degraded because mispoint loss of the main lobe. 

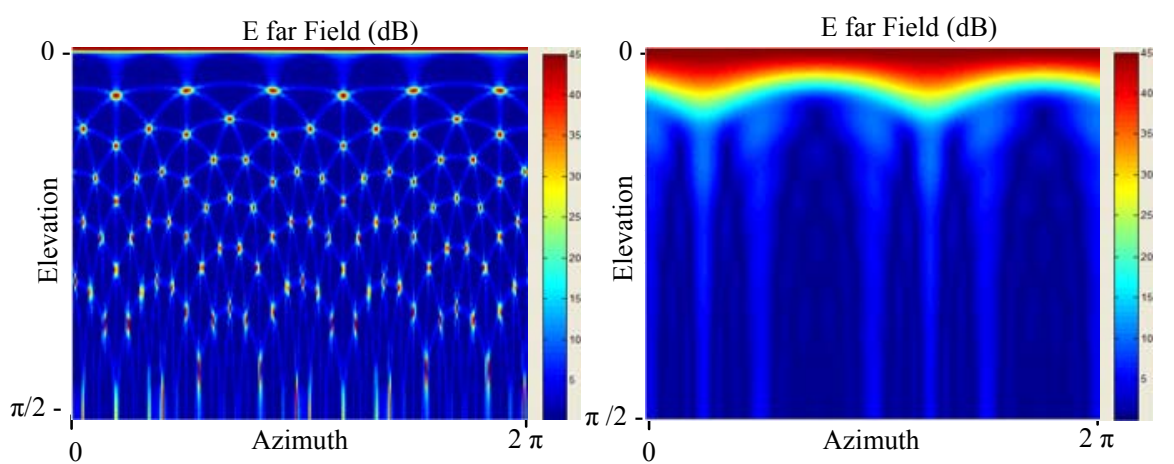

Figure 4. Left: E far field with location error. Right: E far field with ideal sensor location

For a more realistic case a simulation with a sensor location error variance of $0,01027 \lambda$ has been carried out (Figure 5). This location error variance represents the sensor locations error due to the array elements have a non uniform distribution. The side lobe is eliminated with the application of the compensation matrix obtained for the simulated conditions.

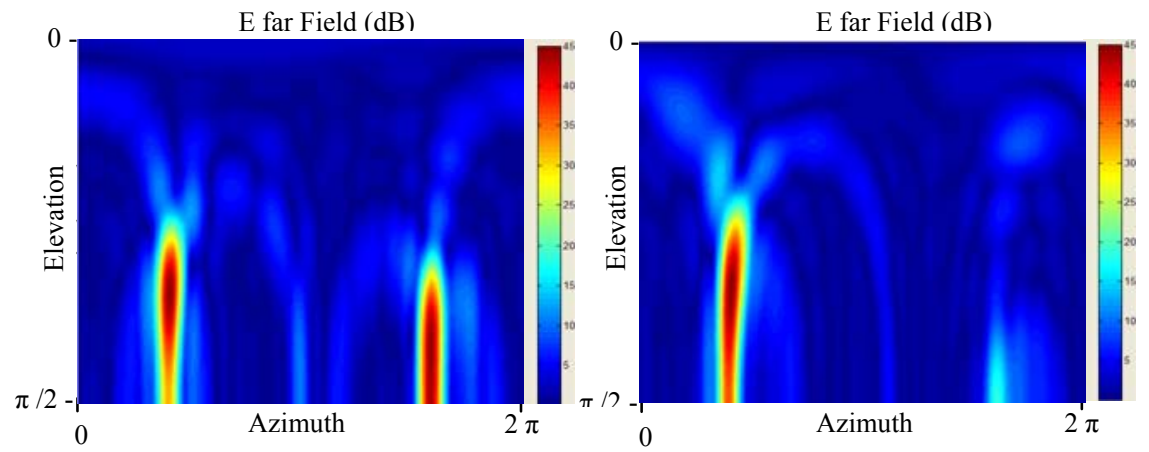

Figure 5. Left: E far field with location error. Right: E far field of the compensated array

\section{Development of the on-line calibration algorithm}

The on-line calibrations algorithms to be evaluated must be transparent. This means that the digital signal processing, beamforming included, must be carried out in parallel task simultaneously.

Since the geometry of the antenna array is well known, a new variable named "Array Entropy" will notify if an element of this array has an unacceptable working condition.

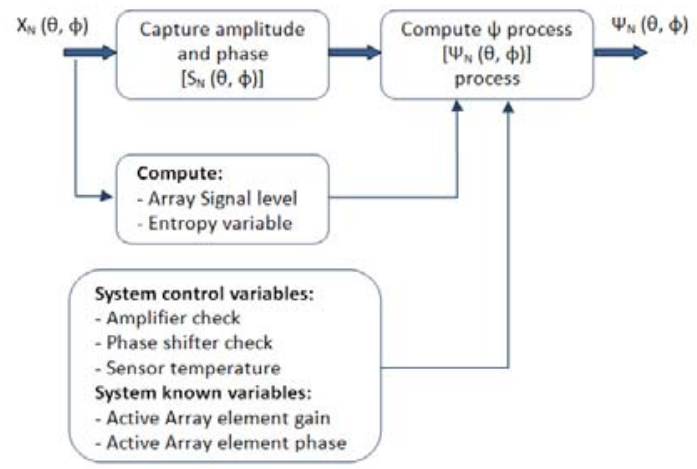

Figure 6. Proposed on-line calibration algorithm 
In the first step of this algorithm (Figure 6), the DoA will be computed with the signal amplitude and phase for each sub-array of $\mathrm{N}_{\mathrm{cc}}$ elements $\left(\mathrm{N}_{\mathrm{cc}}<\mathrm{N}\right)$. Then, a comparison between the calculated DoA $A_{c c}$ with an error threshold of the DoA is carried out. Finally, the sub-array which has the DoA error is selected and it is again subdivided into $\mathrm{N}_{\mathrm{cc}}$ new sub-arrays. The first step is here repeated for these new sub-arrays defined before. As a result, the element which is distorting the steering vector is obtained.

A new compensation matrix for the desired DoA of the Array must be carried out and applied to fix the errors in the array steering vector of the main panel.

\section{Conclusions}

In this contribution, we have presented a preliminary analysis for the implementation of the calibration algorithm to a geodesic antenna array (GEODA) and propose a new algorithm for the on-line calibration. Some simulations was presented in order to allow us explain the scope of this project and the importance of compensate the errors presented at the uncalibrated antenna.

\section{Acknowledgment}

Authors wish to thank MEC (Ministerio de Educación y Ciencia) for the Spanish Government grant (FPI) and the AIMS project (ref: TEC2005-05310/TCM); and INSA (Ingeniería y Servicios Aeroespaciales, S.A.) for the partial funding of this research project.

\section{References}

[1] M. A. Salas, R. Martínez and L. De Haro, "Analysis of antenna array receivers configurations for satellite earth station modems," IEEE International Symposium on Wireless Communication Systems 2007, pp. 313-317, October 2007

[2] A. Torre, J. Gonzalo, R. M. Pulido and R. Martínez, "New Generation Ground Segment Architecture for LEO Satellites," Proc. 57th IAC International Austronautical Congress, Spain, Oct. 2006

[3] M. Sierra-Pérez, A. Torre, J.L. Masa, D. Ktorza and I. Montesinos, "GEODA: Adaptive Antenna Array for Metop Satellite Signal Reception," 4th ESAInternational Workshop on Tracking, Telemetry and Command System for Space Applications, Sept. 2007.

[4] L. C. Godara, "Application of Antenna Arrays to Mobile Communications, Part II: Beamforming and Direction-of-Arrival Considerations," Proc. IEEE, vol. 85, $\mathrm{n}^{\mathrm{o}} 8$, pp. 1195-1245, Aug. 1997.

[5] R. Ertel, Z. Hu, and J. Reed, "Antenna Array Hardware Amplitude and Phase Compensation Using Baseband Antenna Array Outputs," 1999 IEEE Vehicular Technology Conference Proceedings, vol. 3, pp. 1759-1763, 1999.

[6] G. Tsoulos and M. Beach, "Calibration and Linearity issues for an Adaptive Antenna System," 1997 IEEE Vehicular Technology Conference Proceedings, vol. 3, pp. 1597-1600, 1997.

[7] Y. Chen, A. Chang, and H. Lee, "Array Calibration Methods for Sensor Position and Pointing Errors," Microwave and Optical Technology Letters, vol. 26, pp. 132-137, 2000.

[8] C. See, "Sensor array calibration in the presence of mutual coupling and unknown sensor gains and phases," Electronics Letters, vol. 30, pp. 373-374, March 1994. 